\title{
A Single Case Report of a Patient with Bipolar I Disorder: Focusing on EMDR
}

\section{Jae Woo Park*}

Department of General Counseling, The Korea Counseling Graduate University, Hyo-Ryoung Ro Street, Seoul, Korea

"Corresponding author: Jae Woo Park, Department of General Counseling, The Korea Counseling Graduate University, Hyo-Ryoung Ro Street 366, Seoul, Korea, Tel: + 82-10-3894-1597; E-mail: thanotos@kcgu.ac.kr

Rec date: October 16, 2017; Acc date: November 15, 2017; Pub date: November 17, 2017

Copyright: (c) 2017 Park JW. This is an open-access article distributed under the terms of the creative commons attribution license, which permits unrestricted use, distribution, and reproduction in any medium, provided the original author and source are credited.

\begin{abstract}
Objective: The aim of this study was to investigate the effects of Eye Movement Desensitization and Reprocessing (EMDR) on the residual symptoms of bipolar disorder through a single case $A B$ design.

Method: A single-case experimental $A B$ design was carried out using an EMDR intervention with a patient with bipolar I disorder.

Result: Beck Depression Inventory and Young Mania Rating Scale scores for the single patient with bipolar I disorder decreased rapidly during the intervention (A phase), but slightly increased during the intervention withdrawal (B phase).

Conclusion: The single-case experimental design results demonstrated that EMDR reduced residual depressive and manic symptoms in a patient with bipolar I disorder.
\end{abstract}

Keywords: Trauma; Bipolar I disorder; EMDR; Single case report

\section{Introduction}

Post-Traumatic Stress Disorder (PTSD) is a serious mental disorder that causes high levels of decline in function [1]. EMDR is one of the most effective evidence-based psychotherapy techniques for the treatment of PTSD [2]. EMDR is underpinned by the assumption that severe mental disorders do not form through a single traumatic event, as in PTSD, but may form over time after exposure to smaller traumatic events. Disorders that are difficult to treat via conventional psychotherapy (e.g., borderline personality disorders or eating disorders) [3] are disorders that may form after repetitive experiences of small trauma. This assumption is supported by studies demonstrating that EMDR successfully ameliorated symptoms of such a mental disorder. It has been reported that EMDR is effective in treating disorders like personality disorder, depression, and anxiety disorder, but there is a lack of studies investigating the impact of EMDR on patients with bipolar I disorder. However, according to a recent study, when comparing past trauma experience levels between normal subjects, major depressive patients, and bipolar I disorder patients, the bipolar I disorder group had the highest level of trauma experience, followed by the major depressive disorder group [4]. These results suggest that a high level of traumatic experience may affect the symptoms of bipolar disorder. If this hypothesis is true, it would be expected that symptom improvement would occur when an effective intervention for the management of trauma experience, such as EMDR [5], was applied to patients with bipolar disorder. However, the effectiveness of hypothesis-based treatment should first be researched at the level of case reports [6] before being applied to widespread populations of bipolar disorder patients. It is also necessary to apply the intervention to the recent traumatic precipitation factor of bipolar disorder. The purpose of this study was to employ EMDR, an intervention that deals with trauma, using an $\mathrm{AB}$ design for a patient with symptoms of bipolar disorder following a traumatic experience. The hypothesis to be verified was that the residual symptoms would improve when EMDR was provided and that some symptoms would recur when EMDR was withdrawn.

\section{Case Report}

\section{Subject}

One patient who was diagnosed with bipolar I disorder using the DSM-5 and who had been receiving maintenance pharmacotherapy participated in this study.

\section{Measurement}

Beck depression inventory (BDI): In order to evaluate the degree of residual depressive symptoms during EMDR intervention, the BDI was administered once after each session during both the intervention and withdrawal periods.

Young mania rating scales: In order to evaluate the degree of manic symptoms during the EMDR intervention, the YMRS was administered once after each session, for both the EMDR intervention and withdrawal periods.

\section{Procedure}

In this study, we administered EMDR to a patient diagnosed with bipolar I disorder who was receiving medication and was considered to have experienced past trauma. The stabilization effect of ameliorating residual symptoms during maintenance treatment was traced over four 
Page 2 of 2

sessions. After these four sessions, without EMDR intervention, fifty minutes of supportive counseling was provided for four sessions and symptom measures were administered. If the degree of symptom stabilization during the EMDR intervention phase was greater than that during the intervention withdrawal phase, then the EMDR intervention could be deemed effective.

\section{Results}

As EMDR session progressed, the patient's residual depressive and manic symptoms decreased. When the EMDR intervention was withdrawn, there was a slight increase in symptoms by the final measurement period, but symptoms did not return to baseline. This result was displayed for both depressive and manic symptoms. The results are presented in Figure 1.

An in-depth examination of the results showed that the BDI score was 14 at the first measurement, which indicated a subclinical score, and dropped to 7 and 0 at the third and fourth sessions, respectively. The YMRS score was 9 at the first session, stabilized at 2 at the second session, and then decreased to 0 at the third and fourth sessions.

In the withdrawal period, the BDI and YMRS scores were 6 and 3, respectively, suggesting that symptom management was not better than EMDR intervention.

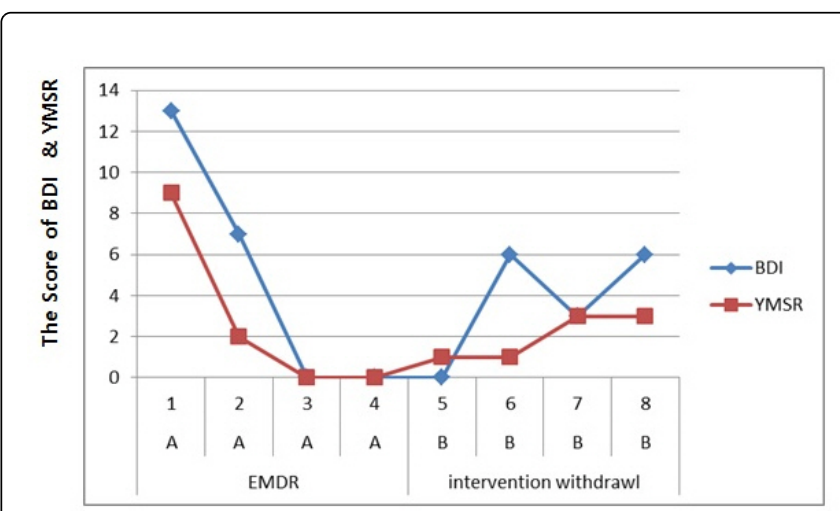

Figure 1: Residual symptom change according to EMDR intervention and withdrawal.

\section{Discussion}

This study assessed the efficacy of an EMDR intervention in a patient with bipolar I disorder. If patients with bipolar disorder have experienced more severe trauma than have those with other disorders, then EMDR may be considered a valid evidence-based psychotherapy intervention, though it must be used alongside medication. Additionally, if this intervention improves symptoms with a single case study, then a larger study would be employed to further ascertain the efficacy of EMDR for the treatment of bipolar disorder.

This study demonstrated that the EMDR intervention reduces depressive and manic symptoms associated with bipolar I disorder. The reduction of symptoms demonstrated here lends support for the efficacy of EMDR as a treatment for patients with bipolar I disorder and who are taking medication. The patient who participated in this study was diagnosed with bipolar I disorder after a stressful experience studying abroad during a mid-term exam period. Therefore, we tailored the EMDR intervention to the patient's stressful experience while preparing for the exam period as well as the conflicting situations of interpersonal relationships experienced in a foreign country.

It is perhaps true that all disorders partly result from a psychosocial stressor-the so-called "precipitating factor." The stressful experience of the patient in study two was considered a type of small psychosocial stressor or trauma, and because it was effective in reducing symptoms, we deem the EMDR intervention an appropriate psychotherapy. According to the findings, the residual depressive and manic symptoms of the patient (who was also under medication for the bipolar I disorder), as measured by BDI and YMRS, were found to be reduced during the four sessions of EMDR. After the withdrawal of the EMDR intervention, the residual symptoms increased slightly but did not return to baseline. This result demonstrates in a single case trial that the EMDR stabilizes manic and depressive symptoms in patients with bipolar I disorder.

\section{Conclusion and Limitation}

This study demonstrated that the patients with bipolar disorder may have experienced more severe repetitive traumatic events than have those in the MDD and healthy control groups.

A limitation of this study is the absence of an ABAB design [6], which would strengthen the study by repeating the conditions to observe ongoing changes in depressive and manic symptoms. However, this study demonstrates that EMDR would be an effective form of psychotherapy for patients with bipolar I disorder and who are taking medication, although it is important to conduct further research with larger sample sizes.

\section{References}

1. American Psychiatric Association (1994) Diagnostic and statistical manual of mental disorders (4th edn) Washington DC, USA.

2. Ursano RJ, Bell C, Eth S, Friedman M, Norwood A, et al. (2004) practice guideline for the treatment of patients with acute stress disorder and posttraumatic stress disorder. Am J Psychiatry 161: 3-31.

3. Shapiro F, Vogelmann-sine S, Sin LF (1994) Eye movement desensitization and reprocessing (EMDR): Treating trauma and substance abuse. J Psychoactive Drug 26: 379-391.

4. Kim HJ, Song W, Park JW (2015) Differences in trauma experience between patients with Bipolar I disorder, patients with major depressive disorder, and healthy controls. J Trauma Dissociation 16: 419-427.

5. Shapiro F (2002) Eye movement desensitization and reprocessing (EMDR) 12 years after its introduction: Past and future research. J Clinic Psychol 58: 1-22.

6. Solanas A, Sierra V, Quera V, Manolov R (2008) Random assignment of intervention points in two-phase single-case designs: Data-divisionspecific distributions. Psychol Rep 103: 499-515. 